\title{
Pulmonary alveolar proteinosis: two contrasting cases
}

\author{
H.L. Crocker*, J. Pfitzner**, I.R. Doyle ${ }^{+}$, W.M. Hague ${ }^{\#}$, B.J. Smith*, R.E. Ruffin*
}

\begin{abstract}
Pulmonary alveolar proteinosis: two contrasting cases. H.L. Crocker, J. Pfitzner, I.R. Doyle, W.M. Hague, B.J. Smith, R.E. Ruffin. (C)ERS Journals Ltd 2000.

ABSTRACT: Pulmonary alveolar proteinosis is a rare condition characterized by the abnormal accumulation of surfactant-like material within the alveolar spaces and distal bronchioles. Two cases with contrasting modes of presentation, course, and response to therapeutic whole lung lavage are described. Both cases were in hypoxaemic respiratory failure at the time the definitive diagnosis was made, and in both cases the diagnosis was made by segmental bronchoalveolar lavage following negative open lung biopsy. In neither was an underlying causative organism or agent identified.

In one case the alveolar proteinosis developed in late pregnancy, a presentation that is previously unreported. Clinical improvement in this case required repeated whole lung lavages and was accompanied by a trend towards normalization of the ratios of surfactant protein-A and surfactant protein-B to disaturated phospholipid, ratios which may be useful as prognostic indicators.

The response to therapeutic lavage was markedly different in the two cases, and it is postulated that this may relate to the fact that alveolar proteinosis is a heterogeneous disease and that the course and response to treatment may relate in part to the specific composition of the abnormal proteinaceous fluid.
\end{abstract}

Eur Respir J 2000; 15: 426-429.

\begin{abstract}
*Respiratory Medicine Unit, **Dept of Anaesthesia, and ${ }^{\#}$ Dept of Obstetrics and Gynaecology, The Queen Elizabeth Hospital, Woodville, South Australia. ${ }^{+}$Dept of Human Physiology, Flinders University of South Australia, Bedford Park, South Australia.
\end{abstract}

Correspondence: H.L Crocker, Respiratory Medicine Unit, The Queen Elizabeth Hospital, Woodville, South Australia 5011, Australia. Fax: 61283713399

Keywords: Alveolar proteinosis pregnancy

pulmonary alveolar proteinosis surfactant

whole lung lavage

Received: October 111998

Accepted after revision May 251999
Pulmonary alveolar proteinosis [1] is a rare condition characterized by the abnormal accumulation of the different components of surfactant within the alveolar spaces [2], with resulting progressive impairment of gas exchange. The disorder is divided into two main categories; primary or idiopathic; and secondary, which occurs in settings of pulmonary infection, immune paresis, including haematological malignancy and human immunodeficiency virus (HIV), and following the inhalation of dusts such as silica and aluminium [3]. The aetiology and pathogenesis of idiopathic alveolar proteinosis remain unclear although evidence increasingly suggests that the presence of defective surfactant homeostasis with impaired removal of surfactant components from the alveolar spaces is more likely than an underlying increase in production [4].

The spectrum of disease is variable, and the prognosis is generally good, frequently with spontaneous remission. However, progressive disease can cause major respiratory dysfunction and can be life threatening. In this event, the only effective treatment is with whole lung lavage [5]. This treatment serves not only to remove from the lung the accumulated surfactant material which interferes with gas exchange, but may also remove a factor or factors which interfere with the physiological mechanisms involved in maintaining normal surfactant homeostasis, or which inhibit alveolar macrophage function [4].

The present report describes two cases of severe pulmonary alveolar proteinosis with contrasting modes of presentation, and markedly differing responses to treatment with whole lung lavage. The content of the lavage fluid from both cases was analysed with reference to the quantity of surfactant-related phospholipids and proteins, and with particular reference to the ratio of surfactant protein (SP)-A to disaturated phospholipid (DSP) since it has been shown that this ratio is high in alveolar proteinosis and may have a diagnostic value [6]. Moreover, SP-A may play a role in surfactant homeostasis, and a lowering of the ratio towards normal may indicate progress towards resolution as suggested in a previously published report on the composition of lavage fluid from 13 cases of alveolar proteinosis which included the two cases presented [7].

\section{Case report}

A 29-yr-old female at 33 weeks of pregnancy was admitted to hospital in acute respiratory distress. She had experienced mild dyspnoea and a dry cough for the preeding 3 months, and in the days prior to admission her dyspnoea and orthopnoea had become extreme. Her cough was productive with occasional scanty white sputum. She was a smoker of 10-20 cigarettes per day, and there were no environmental or occupational exposures of note.

On examination she was cyanosed and tachypnoeic. There was marked peripheral oedema and no adventitial pulmonary sounds on auscultation. An arterial blood gas performed while breathing room air showed an arterial partial pressure of oxygen $\left(\mathrm{Pa}_{\mathrm{a}} \mathrm{O}_{2}\right)$ of $6.0 \mathrm{kPa}(45 \mathrm{mmHg})$, an arterial partial pressure of carbon dioxide $\left(\mathrm{Pa}_{\mathrm{a}} \mathrm{CO}_{2}\right)$ of 4.3 $\mathrm{kPa}(32.6 \mathrm{mmHg})$ and a $\mathrm{pH}$ of 7.45 . Chest radiography demonstrated widespread alveolar opacity, confluent basally. High resolution computed tomography (CT) demonstrated diffuse alveolar opacity with a "crazy paving" 
configuration extending throughout both lung-fields but sparing some areas, particularly in the lung periphery and at both the base and the apex of the lung (fig. 1).

A presumptive diagnosis of pulmonary alveolar proteinosis was made, supplemental oxygen therapy was instituted, and corticosteroid therapy was administered to assist in foetal lung maturation. Empirical high dose prednisolone and broad spectrum antibiotic therapy were also commenced pending confirmation of the diagnosis.

Six hours after admission to hospital an emergency Caesarean section and an open lung biopsy were performed under general anaesthesia. After surgery the patient was maintained on intermittent positive pressure ventilation (IPPV). The baby was transferred to a neonatal intensive care unit with a diagnosis of hyaline membrane disease.

The lung biopsy, which was taken from an area of lung perceived on the CT scan to contain infiltrate extending toward the lung periphery, was found at initial and at subsequent re-examination to be within normal limits. The surface of the lung and the biopsy specimen appeared macroscopically normal.

Fibreoptic bronchoscopy was performed via the endotracheal tube, and right middle lobe lavage with $50 \mathrm{~mL}$ aliquots of normal saline returned macroscopically turbid fluid. Light microscope examination revealed periodic acid-Schiff (PAS) positive amorphous proteinaceous material that was found on electron microscopy to contain lamellar bodies pathognomonic for pulmonary alveolar proteinosis.

Twenty-seven hours after the Caesarean section and lung biopsy, a whole lung lavage was performed on first the left lung, the same side as the open lung biopsy. During the procedure, the endotracheal tube was replaced with a double-lumen endobronchial tube and 25 one litre volumes of normal saline buffered with sodium bicarbonate to a $\mathrm{pH}$ of $\sim 7.4$ and warmed to $37^{\circ} \mathrm{C}$, were run into and drained out of the left lung. The right lung was lavaged 3 days later.

The patient remained intubated and on IPPV over this period. After both lungs had been lavaged, the patient's oxygenation improved, and she was able to be extubated and transferred to the thoracic ward on supplementary oxygen.

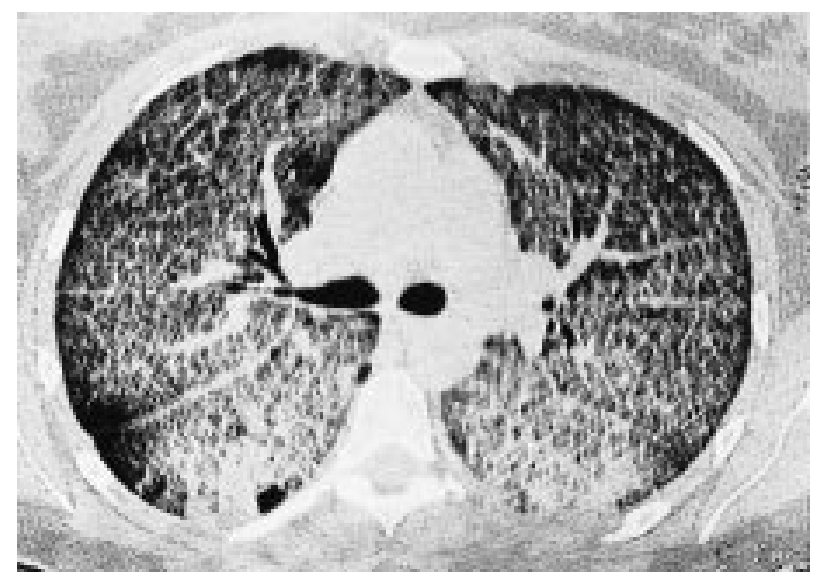

Fig. 1. - Computed tomographic scan of case 1 showing diffuse changes consistent with pulmonary alveolar proteinosis (PAP). Despite targeting the involved areas at open lung biopsy, the histopathological examination was negative for PAP
Four further whole lung lavages were performed before the patient was well enough, on day 37 of her admission, to be discharged on home oxygen therapy.

Following discharge the patient was monitored closely as an outpatient, with periodic arterial blood gases, chest radiographs, pulmonary function tests, and exercise oximetry. The $\mathrm{Pa}_{2} \mathrm{O}_{2}$ on room air remained $<6.7 \mathrm{kPa}(<50$ $\mathrm{mmHg}$ ) for in excess of 12 weeks, and hypoxaemia and recurring symptoms necessitated a further eight whole lung lavages at increasing intervals. The final and 14th lavage was performed 58 weeks after the initial presentation. Over this time $\mathrm{Pa}, \mathrm{O}_{2}$ breathing air increased from $6.0 \mathrm{kPa}(45$ $\mathrm{mmHg}$ ) to $8.5 \mathrm{kPa}(64 \mathrm{mmHg})$, and the carbon monoxide diffusing capacity of the lung $(D \mathrm{~L}, \mathrm{CO})$ improved progressively from $<40 \%$ to $90-100 \%$ of predicted value. Twelve months following the last lavage the patient was symptomfree with normal pulmonary function tests, and was riding her exercise bicycle 50 kilometres a day.

Lavage fluid collected from the first whole lung lavage was sent for comprehensive examination for possible causative organisms or agents, cytology, and cell counts with differential estimations. The relative amounts of the different components of surfactant were measured in the last 11 lavage samples.

As the clinical picture improved with time and further lavages, the ratio of SP-A to DSP fell progressively towards reported normal values, as did the ratio of SP-B to DSP and to a lesser extent the ratio of cholesterol (Cholest) to DSP [7]. In table 1, the SP-A/DSP ratio and the Cholest/DSP ratio in lavage fluid from the 4th and 5 th lavages, which were the first two lavages analysed in detail, and also from the 13th and the final 14th lavages are shown, together with the ratio of DSP to total phospholipid (PL) and the SP-B/SP-A ratio. The mean "normal" values obtained from right middle lobe bronchial lavage of 16 healthy subjects [7], and the values for Case 2 are also presented.

Table 1. - Relative concentrations of surfactant-related proteins and lipids in lavage fluid collected from the 4th and 5th and also from the 13th and 14th lavages which were performed on Case 1, from the two lavages on Case 2 , and from healthy subjects

\begin{tabular}{|c|c|c|c|c|}
\hline & SP-A/DSP & $\begin{array}{c}\text { Cholest/ } \\
\text { DSP }\end{array}$ & DSP/PL & SP-B/SP-A \\
\hline $\mathrm{C}$ & $0.15 \pm 0.01$ & $0.12 \pm 0.01$ & $0.49 \pm 0.01$ & $2.27 \pm 0.19$ \\
\hline $\begin{array}{l}\text { Lung } \\
\text { Case } 1\end{array}$ & $\mathrm{R} \quad \mathrm{L}$ & $\mathrm{R} \quad \mathrm{L}$ & $\mathrm{R} \quad \mathrm{L}$ & $\mathrm{R} \quad \mathrm{L}$ \\
\hline 4 th $+5 \mathrm{th}^{\# *}$ & 4.075 .18 & 0.720 .70 & $\begin{array}{lll}0.80 & 0.43\end{array}$ & 1.861 .74 \\
\hline $13 \mathrm{th}+14 \mathrm{th}^{\#}$ & 1.161 .22 & 0.420 .48 & 0.510 .61 & 2.351 .46 \\
\hline Case 2 & 6.002 .70 & 0.740 .72 & 0.410 .42 & 2.444 .95 \\
\hline
\end{tabular}

Data are presented as absolute numbers and mean \pm SD. SP-A/ DSP: ratio of surfactant protein-A to disaturated phospholipid; Cholest/DSP: ratio of cholesterol to disaturated phospholipid; DSP/PL: ratio of disaturated phospholipid to total phospholipid; SP-B/SP-A: ratio of surfactant protein-A to surfactant protein-B; $\mathrm{C}$ : control subjects; R: right; L: left. " ${ }^{\#}$ lavages; *: a detailed analysis was not conducted on lavage fluid obtained from the 1 st, 2nd and 3rd lavages. 


\section{Case 2}

A 34-yr-old male with a 15 pack-year smoking history presented to a thoracic specialist for investigation of progressive exertional dyspnoea over recent months. He had a past medical history of depression, longstanding lower back pain with consequent narcotic dependency, peptic ulcer disease, and an undefined syndrome of episodic angioedema and urticaria. There were no occupational or environmental exposures of note.

He complained of dyspnoea on exertion and a dry cough, generalized aches and pains, and reported progressive disability. He was clubbed, which was claimed to be familial, and auscultation of the chest revealed sparse bibasal crackles, which were not consistently present.

Initial investigations were suggestive of possible early interstitial lung disease, though a full diagnostic work-up was hampered by poor patient compliance with investigations and the involvement of a number of different medical practitioners such that he was commenced on moderately high dose prednisolone prior to definitive diagnosis.

He developed a nocardial lung abscess in the left upper lobe, which was treated with a protracted course of trimethoprim/sulphamethoxazole, and sputum samples grew Mycobacterium Avium intracellulare, which was monitored clinically and without specific intervention.

His condition deteriorated over a period of 4 months while on prednisolone therapy, with progressive dyspnoea and hypoxaemia. An arterial blood gas performed whilst breathing room air showed a $\mathrm{Pa}, \mathrm{O}_{2}$ of $5.4 \mathrm{kPa}(41 \mathrm{mmHg})$ and a $P \mathrm{a}, \mathrm{CO}_{2}$ of $4.3 \mathrm{kPa}(32 \mathrm{mmHg})$; pulmonary function testing revealed a moderate restrictive deficit and a marked reduction in the transfer factor; and high resolution $\mathrm{CT}$ scans showed diffuse ground glass opacification throughout most of the lung fields (fig. 2). An open lung biopsy was performed with due regard to the CT appearances, but was negative.

Four weeks after the open lung biopsy his condition had deteriorated further, culminating in hypoxaemic respiratory failure requiring intensive care unit admission for intubation and IPPV. A repeat open lung biopsy, and fibreoptic bronchoscopy with segmental bronchoalveolar lavage, were performed and both proved diagnostic of alveolar proteinosis.

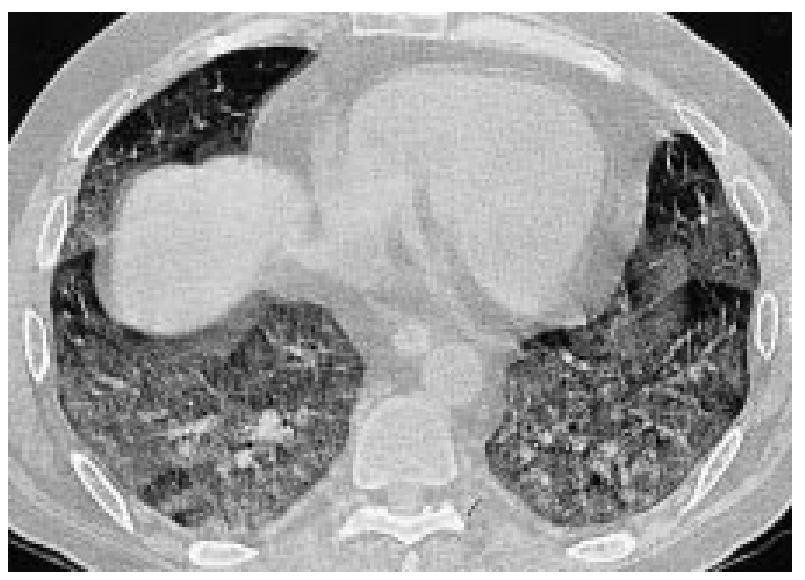

Fig. 2. - Section from the computed tomographic scan of case 2 showing changes typical of pulmonary alveolar proteinosis. Despite the diffuse involvement, the open lung biopsy was negative for pulmonary alveolar proteinosis.
Sequential whole lung lavage was undertaken on first the left lung and 3 days later the right lung. At the time of the first lavage the patient had been on IPPV for 5 days with a $P \mathrm{a}, \mathrm{O}_{2}$ ranging $7.7-10.4 \mathrm{kPa}(58-78 \mathrm{mmHg})$, on an inspiratory oxygen fraction $\left(F \mathrm{I}, \mathrm{O}_{2}\right)$ of $0.75 \mathrm{kPa}$ and a positive end-expiratory pressure (PEEP) of $0.5 \mathrm{kPa}$. The lavage effluent was subjected to comprehensive examination and was also analysed for the different surfactant components (table 1). Improvement was dramatic following lavage of the right lung, enabling weaning from ventilation 4 days later, transfer to the ward, and subsequent discharge 2 weeks later to a nursing home for further rehabilitation for back pain and muscular wasting.

When last seen 15 months after diagnosis, the patient was free of respiratory symptoms and there were no indications for further lavage. Serial follow-up pulmonary function tests were essentially normal with a minor residual decrease in the diffusion factor, and arterial blood gases on air showed a $P \mathrm{a}_{0} \mathrm{O}_{2}$ of $11.0 \mathrm{kPa}(83 \mathrm{mmHg})$, an oxygen saturation of $96 \%$, and a $P \mathrm{a}_{2} \mathrm{CO}_{2}$ of $5.3 \mathrm{kPa}(40$ $\mathrm{mmHg}$ ).

\section{Discussion}

The two cases of pulmonary alveolar proteinosis reported highlight the wide spectrum of this disease process, with both differing modes of presentation and differing response to treatment with therapeutic whole lung lavage.

One case first presented in late pregnancy with the precipitous onset of respiratory failure following a minor prodrome of dry cough and breathlessness over three months. Alveolar proteinosis presenting in pregnancy has not been previously reported, and treatment with 14 therapeutic lavages over a period of 58 weeks was required before a sustained response was achieved. Since surfactant homeostasis is altered by cytokines and growth factors including granulocyte-macrophage colony-stimulating factor (GM-CSF) [8] and keratinocyte growth factor (KGF) [9], it is tempting to speculate on an association between changes in the maternal levels of these factors during pregnancy and post-partum, and the development and subsequent resolution of the condition. However, we have no way of substantiating this.

The second case presented insidiously over a period of at least 6 months, and responded immediately to a single therapeutic lavage of each lung performed 3 days apart.

Both patients were in hypoxaemic respiratory failure at the time of diagnosis, and required IPPV at the time of their first whole lung lavages. The lavage procedure was well tolerated in both patients.

Of interest is the fact that the initial lung biopsy was nondiagnostic in both cases. The patchy nature of pulmonary alveolar proteinosis and the usually lesser involvement of the lung periphery renders a negative biopsy result possible, and suggests that open biopsy is not the diagnostic gold standard. The less invasive procedure of segmental bronchoalveolar lavage [10] of the right middle lobe using four $50 \mathrm{~mL}$ aliquots of normal saline proved diagnostic in both cases.

Whole lung lavage following recent open lung biopsy is not without theoretical risk, and it is considered advisable that the initial lavage is performed on the side of the biopsy 
so that the vitally important single-lung ventilation is performed on the lung which has not been biopsied.

The composition of the lavage fluid was analysed for the different surfactant components in both cases (table 1), and in case 1 the concentration of the different components was shown to fall progressively during the course of each lavage [7] and also to differ between the separate therapeutic lavages as the disease responded to treatment (table 1).

Pulmonary alveolar proteinosis is a heterogeneous disease, and it is tempting to speculate that this may be either the cause or the effect of the differing composition of fluid obtained during therapeutic lavages in the two cases presented. Further study of the composition of lavage fluid is indicated as this could conceivably be linked to disease course. Since the ratio of SP-A and SP-B to DSP returned towards normal levels as the clinical condition of case 1 improved and the requirement for therapeutic lavage abated [7], the analysis of the content of lavage fluid from repeated therapeutic whole lung lavages may prove to be a prognostic indicator in predicting response to treatment or progress towards resolution.

In conclusion, there is much that is not known about the pathogenesis and resolution of alveolar proteinosis. Although both the cases presented were in hypoxaemic respiratory failure and required intermittent positive-pressure ventilation at the time of diagnosis, one had presented insidiously but responded to a single bilateral whole lung lavage, while the other presented acutely but required repeated lavages before improvement was maintained. It is postulated that the differences in presentation and response to treatment may relate in part to the specific composition of the abnormal proteinaceous fluid.

\section{References}

1. Rosen SH, Castleman B, Liebow AA. Pulmonary alveolar proteinosis. N Engl J Med 1958; 258: 1123-1142.

2. Singh G, Katyal SL, Bedrossian CW, Rogers RM. Pulmonary alveolar proteinosis. Staining for surfactant apoprotein in alveolar proteinosis and in conditions simulating it. Chest 1983; 83: 82-86.

3. Wasserman K, Mason GR. Pulmonary alveolar proteinosis. In: Murray JF, Nadel JA, eds. Textbook of Respiratory Medicine. 2nd Edn. Philadelphia, USA, WB, Saunders Company, Philadelphia, 1994; pp. 1933-1946.

4. Alberti A, Luisetti M, Braschi A, et al. Bronchoalveolar lavage fluid composition in alveolar proteinosis. $\mathrm{Am} \mathrm{J}$ Respir Crit Care Med 1996; 154: 817-820.

5. Selecky PA, Wasserman K, Benfield JR, Lippmann M. The clinical and physiological effect of whole-lung lavage in pulmonary alveolar proteinosis: a ten year experience. Ann Thorac Surg 1997: 24: 451-461.

6. Honda Y, Takahashi H, Shijubo N, Kuroki Y, Akino T. Surfactant protein-A concentration in bronchoalveolar lavage fluids of patients with pulmonary alveolar proteinosis. Chest 1993; 103: 496-499.

7. Doyle IR, Davidson KG, Barr HA, Nicholas TE, Payne K, Pfitzner J. Quantity and structure of surfactant proteins vary among patients with alveolar proteinosis. Am J Respir Crit Care Med 1998; 157: 658-664.

8. Dranoff G, Crawford AD, Sadelain M, et al. Involvement of granulocyte-macrophage colony-stimulating factor in pulmonary homeostasis. Science 1994; 264: 713-716.

9. Ikegami M, Jobe AH, Havill AM. Keratinocyte growth factor increases surfactant pool sizes in premature rabbits. Am J Respir Crit Care Med 1997; 155: 1155-1158.

10. Martin RJ, Coalson JJ, Rogers RM, Horton FO, Manous LE. Pulmonary alveolar proteinosis: the diagnosis by segmental lavage. Am Rev Respir Dis 1980; 121: 819825 . 